\title{
Aplicaciones de la Evaluación Comparativa: el estado de la cuestión
}

\section{The Benchmarking applications: a critical review}

\author{
Jesús Manuel Plaza Llorente \\ Universidad Nacional de Educación a Distancia (UNED). Facultad de Ciencias Económicas y Empresariales. \\ P.\%/ Senda del Rey, II. 28003 Madrid jplaza@cee.uned.es
}

Fecha de recepción: 08-07-2010

Fecha de aceptación: 03-12-2010

Resumen: Después de medio siglo la Evaluación Comparativa o Benchmarking está presente en multitud de evaluaciones que hacen uso de ella en alguna de sus fases. A partir de este hecho, el artículo aborda una revisión de sus posibilidades en los dominios organizativo y empresarial. Tras definir un marco de análisis, se revisa la evolución histórica de las aplicaciones de la evaluación comparativa en diversos ámbitos, tomando como referencia trabajos publicados. Por último, se realiza un juicio crítico sobre el uso actual del benchmarking como herramienta metodológica para la práctica profesional de la evaluación de políticas públicas, organizativas y de empresa.

Palabras clave: Evaluación comparativa, evaluación de políticas, aprendizaje.

Abstract: After fifty years of use of Benchmarking, this tool of analysis has been incorporated in a large number of professional works of organizational and enterprise evaluation. On this fact, this paper makes an historical review of the applications of this tool emphasizing its possibilities. After the methodological framework and definitions, the article summarizes the benchmarking role in research. Before the conclusions, a chapter is dedicated to make a critical judgment of benchmarking applications towards the professional practice for public, organizational and enterprise policies evaluation.

Key words: Benchmarking, policies evaluation, benchlearning.

\section{Introducción}

Constituye el objeto de este artículo revisar las aplicaciones que ha tenido la Evaluación Comparativa o benchmarking durante el último medio siglo, desde su uso en el dominio industrial hasta su generalización para el moderno análisis de políticas públicas. También es una investigación teórica en torno a este concepto, aspecto éste poco tratado por la literatura académica y considerado clave por Intxaurburu y Ochoa (2005).

Hoy, el benchmarking aparece como una herramienta analítica consolidada para la evaluación en los ámbitos organizativo y empresarial que permite su uso en multitud de investigaciones y con fines muy diversos (Comisión Europea 2000b, 2003, Keegan 2000b).

En los primeros años ochenta del siglo pasado científicos, consultores y decisores públicos y privados encontraron en la evaluación comparativa una herramienta que, por su simplicidad conceptual y aparente facilidad de uso, ponía a su alcance la dirección del programa o la gestión de la organización con sólo mirar un cuadro de indicadores. Dicho cuadro sólo debería ser representativo del estado de la realidad económica u organizativa que era objeto de investigación con respecto a otra líder, y cuya pauta de funcionamiento no habría más que seguir (Watson 1993, Boxwell 1994).

Durante la década de los noventa del siglo $X X$, el benchmarking se implantó de forma generalizada como método de análisis en los organismos internacionales más prestigiosos y, curiosamente, a medida que su utilización era mayor dejó de hablarse de él; como síntoma de haberse incorporado de forma inequívoca al corpus teórico metodológico y al bagaje práctico de la gestión empresarial y de programas sociales.

En el terreno de la evaluación de programas y políticas públicas (OCDE 2005), más recientemente el benchmarking ha protagonizado procesos que tuvieron trascendencia histórica, como fue la adopción de la moneda única por la Europa comunitaria o que pasaron a consolidar prácticas en la evaluación de rea- 
lidades complejas que, de forma sucesiva, han ido incorporándose a la agenda internacional desde la lucha contra la pobreza hasta el cambio climático (OCDE 2010).

Tras esta presentación, en los capítulos primero y segundo del artículo se definen la metodología seguida para su redacción y los conceptos fundamentales en torno a los que se construye el análisis. Un tercer capítulo sirve para establecer el alcance científico del benchmarking como método de investigación para, en el cuarto capítulo, llevar a cabo una revisión histórica de sus aplicaciones desde que comenzara a utilizarse en ingeniería como instrumento formalizado de conocimiento.

En el quinto capítulo se lleva a cabo una reseña de las principales aplicaciones actuales del benchmarking en ámbitos económicos y organizativos relevantes. Antes de formular las conclusiones, en el sexto capítulo de este trabajo se presenta un juicio crítico del benchmarking como herramienta metodológica, señalando cómo puede contribuir a enriquecer la práctica profesional de la evaluación y análisis de las organizaciones, los programas empresariales y las políticas públicas!.

\section{Metodología}

Para revisar la evolución temporal y actualizar las referencias sobre el benchmarking, este trabajo ha hecho uso de Internet desde una doble perspectiva. En primer término para señalar publicaciones y sitios web a los que pertenecen textos que han sido objeto de consideración. Como segunda posibilidad, y utilizando el buscador Google 2 como herramienta exploratoria, para cuantificar la relevancia del benchmarking en ámbitos sectoriales y temáticos.

Si hasta el inicio de la década de los ochenta del siglo pasado el uso de la evaluación comparativa se realizaba fundamentalmente en el ámbito de la gestión económica, esta investigación nos permitirá conocer cómo y por qué se ha extendido su aplicación a otros ámbitos. Para ello se ha adoptado el enfoque sumativo de Scriven (1980) que permite observar la evolución del benchmarking como herramienta de evaluación, orientada a medir y valorar organizaciones e instrumentos de planificación mediante indicadores cuantitativos, con el objetivo de proporcionar información útil para la gestión.

\section{Definiciones}

En primer lugar se fija la de benchmarking como objeto de estudio, para precisar después el alcance de cinco conceptos en torno a los que se han producido los desarrollos más novedosos de la evaluación comparativa en el pasado reciente: el conocimiento, la información, la innovación, la gestión del talento y las políticas públicas.

La elección de estos cinco ámbitos no ha sido casual sino producto de esta misma investigación por cuanto, al analizar el tránsito histórico de las aplicaciones de la evaluación comparativa, se constata la existencia de usos recurrentes que llegan hasta hoy vinculados a estos cinco aspectos fundamentales del progreso empresarial y organizativo.

\section{I. Benchmarking o evaluación comparativa}

La expresión Evaluación Comparativa o Benchmarking tuvo sus primeras definiciones en el dominio industrial en trono a 1975, cuando importantes acerías norteamericanas, japonesas y europeas comenzaron a analizar en paralelo sus procesos comerciales y de fabricación con fines de aprendizaje (Allen 1996). De esta manera, el benchmarking apareció como un método de comparación sistemática formalizado que pretendía mejorar los resultados económicos de las empresas participantes en su aplicación, a través de identificar una o varias Mejores Prácticas ${ }^{3}$ susceptibles de implantarse en tales organizaciones (Bogan y English 1994).

Sin embargo, fue la experiencia de Xerox Corporation en 1979 y su responsable de logística Robert Camp los que pautaron la evaluación comparativa como proceso destinado a la búsqueda de las mejores prácticas a partir del comportamiento de una organización particular que, de acuerdo con uno o varios indicadores, se consideraba líder (Camp 1989).

\footnotetext{
' Entendemos por Evaluación: «el análisis normalizado y sistemático de la información relativa a un programa o intervención pública, con el objetivo de emitir un juicio fundado y con carácter de rendición de cuentas, que permita a los Grupos de Interés (stakeholders) tener una idea clara y distinta sobre la utilidad, pertinencia y adecuación de la acción evaluada a su contexto».

2 http://www.google.es/

3 La Comisión Europea (2000a) define Mejor Práctica como: «la superior capacidad de gestión demostrada para una función particular en la industria, el liderazgo en la dirección o los métodos operativos utilizados, que conducen a una empresa u organización a obtener resultados excepcionales».
} 
Spendolini (1994) define la evaluación comparativa como «un proceso sistemático y continuo para evaluar productos, servicios y procesos de trabajo en las organizaciones que son reconocidas como representantes de las mejores prácticas con el propósito de realizar mejoras organizativas».

Para el Departamento de Comercio e Industria del Reino Unido, el Best Practice Benchmarking (BPB) constituía en 1998 una técnica destinada a aprender de las organizaciones que destacaban en determinados aspectos de gestión. Dicho aprendizaje se abordaba a través de cuatro etapas: I) identificar los factores o elementos clave que caracterizaban la obtención de mejores resultados por una organización líder, 2) establecer indicadores de medida de los factores o elementos identificados que se consideraban clave, 3 ) investigar por qué las organizaciones líderes alcanzaban mejores resultados que las demás y, 4) establecer y aplicar planes de acción en las organizaciones cuyos indicadores mostraban un potencial de mejora con respecto a los del líder.

La idea de Proceso, sustancial para la evaluación comparativa, se ve reforzada en la definición que hace de ella la Comisión Europea (2000a), al considerarla como un «proceso sistemático y continuo de medida y comparación de la gestión de una organización con respecto a la de organizaciones líderes en cualquier parte del mundo». También, el término japonés dantotsu (lo mejor de lo mejor) sirve a Camp (2000) para aprehender la esencia del benchmarking como «proceso, positivo y proactivo destinado a cambiar el modo en que se estructuran las acciones de una organización para lograr una mejor gestión, con el objetivo de alcanzar una ventaja competitiva». Por último, la consideración que la evaluación comparativa merece al Instituto Tecnológico Danés (Keegan 2000a) es la de proporcionar una «caja de herramientas» simple, accesible y eficiente; capaz de ayudar a la organización a identificar áreas con potencial de mejora para su competitividad, productividad y crecimiento.

Cualquiera de las definiciones anteriores permite encontrar en el benchmarking la presencia de los elementos que caracterizan la Evaluación: orientación a un fin, análisis de una situación-problema, presencia de una sistemática en el proceso, existencia de un juicio valorativo y enfoque utilitario. Además, esta posibilidad, la de que el benchmarking fuera una modalidad evaluativa, permitiría superar dificultades terminológicas como las manifestadas por Intxaurburu y Ochoa (2005). Sin embargo, antes de afirmar a la evaluación comparativa como categoría o modalidad de evaluación, conviene seguir a Spendolini (1994) para señalar lo que no es benchmarking.

En primer lugar, el benchmarking no responde a un hecho aislado en la vida de la organización sino que se inserta en un proceso de mejora continua que, cómo es sabido, no es rápido ni sencillo. En segundo término la evaluación comparativa no consiste en copiar; sino en saber porqué otra organización hace lo mismo mejor, a partir de establecer determinados criterios que permitan cuantificar ese nivel superior de desempeño o de gestión. En tercer lugar, y como sucede tantas veces, el benchamarking no debería responder a una moda sino ser el resultado de una opción de futuro en la relación de una organización con su entorno. Finalmente, de la evaluación comparativa no deben esperarse recetas sino, por el contrario, la identificación de factores limitantes para acometer planes de mejora organizativa coherentes que respondan a objetivos concretos y mensurables.

\subsection{Conocimiento}

El Conocimiento lo encontramos asociado en nuestros días a la existencia de una sociedad (llamada del conocimiento) en la que la investigación científica, las tecnologías de las comunicaciones y el acceso generalizado de las personas a los contenidos informativos nos han situado ante un salto schumpeteriano.

\subsection{Información}

Para el proceso de benchmarking la información constituye un recurso principal y garantía de éxito según sea su calidad y fiabilidad. En este sentido, Denrell (2003) ha señalado el problema que los sesgos y la deficiente calidad de la información estadística suponen para la credibilidad final de un proceso de evaluación comparativa.

\subsection{Innovación}

En el dominio empresarial y organizativo la innovación se materializa como la «creación o modificación de un producto junto a su introducción con éxito en el mercado» (Molero 200 I). Para Drucker (1986) la importancia de la innovación se mide a partir de las necesidades que satisface, procedan éstas de un cliente o de un agente social, siendo frecuente su caracterización mediante indicadores (Hidalgo,Vizán yTorres 2008). 


\subsection{Gestión del talento}

Más recientemente ha sido Peters (2002) el que ha puesto al día el papel que juega el talento en las organizaciones en cuanto que capacidad para ejercitar una tarea con inteligencia. De hecho, gran parte del trabajo de Peters como teórico y gurú del pensamiento organizacional de nuestros días, tuvo su origen en la práctica profesional del benchmarking como método de análisis en consultoría estratégica. Este mismo autor atribuye al talento capacidad para crear imagen de marca con fines competitivos puesto que «en el mercado internacional ya no son los productos los que compiten, sino los conceptos»».

\subsection{Políticas públicas}

En cuanto a las Políticas o Intervenciones Públicas, las entenderemos en dos de las acepciones que propone McLean (1996). La primera de ellas es la de Política, como expresión de la práctica del arte o ciencia de dirigir o administrar los estados u otras entidades políticas, tales como las organizaciones. La segunda, será la de Economía Política o rama del arte de gobernar, interesada por la investigación sistemática de la naturaleza y causas de la riqueza y bienestar.

\section{Alcance del benchmarking como método científico y de evaluación}

A partir de su definición la evaluación comparativa aparece como un método de análisis que permite separar las partes de un todo, para poner éstas junto a otras de realidades parecidas, con el objetivo de observar qué diferencias existen entre ellas. Por tanto, en el plano espistemológico el benchmarking opera o actúa mediante comparación o cotejo y razona mediante la analogía, para concluir si las realidades comparadas son semejantes o no.

En la valoración del alcance de la evaluación comparativa como método de hacer ciencia, podríamos preguntarnos si es capaz por sí misma de desarrollar el método científico completo, si lo hace de forma incompleta o si precisa de herramientas auxiliares. El paradigma científico de nuestros días establece cuatro etapas: I) observación de los fenómenos investigados, 2) formulación de hipótesis explicativas de tales fenómenos, 3) falsación de las hipótesis mediante mé- todos aceptados, lo que conducirá a aceptarlas o rechazarlas concediéndolas valor de verdad, y 4) incorporación de las hipótesis aceptadas o negadas al cuerpo teórico o axiomático de la ciencia en cuestión para su uso con fines predictivos de otras realidades o para, en general, abordar otras investigaciones.

El benchmarking aborda la primera de las etapas del método científico (Observación) mediante la exposición sistemática de las realidades objeto de comparación a través de variables cuantificadas, susceptibles de cuantificación indirecta (proxy) o mediante relaciones ordinales o lógicas («superior a» o «preferido a»). Dicho en otras palabras; la evaluación comparativa descompone la realidad observada y la caracteriza mediante variables que la representan, haciendo lo propio con las otras realidades que entran en la comparación mediante el uso de las mismas variables.

A partir de tal exposición formal, y con el auxilio del acervo teórico de la materia investigada, el benchmarking puede formular hipótesis acerca del comportamiento de las variables producto de la comparación y de sus relaciones entre ellas (conexión o correspondencia) o concomitancias (acompañamiento o cooperación).

Por sí misma la evaluación comparativa no posee capacidad de inferencia, realizable mediante falsación popperiana, que permita conferir valor de verdad a las tesis (hipótesis no demostradas) de relación o concomitancia entre las variables. Para ello requiere del concurso de pruebas de hipótesis que permitan realizar la inferencia o deducción en cuanto que elemento capaz de proporcionar validez científica al razonamiento (Martínez 1993).

Finalmente, $y$ en el supuesto de haber podido falsar las tesis con arreglo a métodos de general aceptación por la comunidad científica, las deducciones procedentes del benchmarking son susceptibles de incorporarse al cuerpo teórico de las ciencias, de la misma forma que lo son las hipótesis formuladas por métodos axiomáticos o reductivo-inductivos una vez sometidas al método científico.

Por tanto, a la pregunta de si a través del razonamiento analógico utilizado por el benchmarking es posible crear ciencia, hay que contestar afirmativamente; siempre que se cuente con el concurso auxiliar de un procedimiento aceptado ${ }^{4}$ que permita

\footnotetext{
${ }^{4}$ Los «procedimientos aceptados» lo son por la comunidad de cada rama científica. Para muchas de ellas este procedimiento lo constituyen las pruebas de hipótesis, en otros casos lo será la reproductividad del fenómeno en el laboratorio o en la realidad social y, en otros, la validación de modelos lógicos, matemáticos o econométricos.
} 
falsar las tesis investigadas procedentes de la comparación.

\section{I. La evaluación comparativa en las ciencias de la organización}

La Comparación o el Método Comparativo tiene una larga tradición en las ciencias sociales y de la organización desde que Stuart Mill lo propusiera en su A System of Logic, estableciendo los cánones de concordancia, diferencia, residuos y variaciones concomitantes y que hizo que Durkeheim lo afirmase «como el único que conviene a la sociología» (Beltrán 1985). Este recurso a la comparación sistemática de los fenómenos puede tener carácter bien transversal (cross section) bien longitudinal; de la misma manera que en el ámbito al que se aplique es susceptible de enfoques macro o micro, como sucede en la economía.

En consecuencia se puede afirmar la validez de los criterios generales de clasificación de las investigaciones científicas cuando se realizan bajo las modalidades de benchmarking, de la misma forma que su utilidad como método para el científico y el gestor, pues «sólo cuando puedes medir aquello de lo que estás hablando y expresarlo con números, sabes algo acerca de ello; pero cuando no puedes medirlo, cuando no puedes expresarlo con números, tu conocimiento es pobre e insatisfactorio», en frase de Lord Kelvin.

La rotundidad de la frase del físico y matemático Kelvin, empleada aquí como argumento de autoridad sobre el valor intrínseco de lo cuantificable y (por tanto) de la evaluación comparativa cuando trabaja con variables cuantitativas, no debería suscitar ni poner en duda el hecho de que, en nuestros días, los métodos de investigación cualitativos y cuantitativos forman parte de la pauta o sistemática de cualquier investigación. La experiencia señala que toda investigación llevada a cabo mediante benchmarking hace uso de ambos tipos de procedimientos y que, desde el punto de vista metodológico, lo que diferencia a unas investigaciones de otras es la proporción con la que las herramientas cualitativas y cuantitativas contribuyen a conseguir los objetivos científicos, técnicos o de gestión.

En el terreno de la ciencia política, Mckenzie (1972) otorga a la comparación carta de naturaleza por cuanto la considera «instrumento necesario de des- cubrimiento», al punto de que «el problema no constituye en decidir si hay que comparar, sino en cómo organizar la comparación».

Con el desarrollo de los sistemas de indicadores (Rossi y Freeman 1982), la evaluación comparativa se ha utilizado más recientemente como herramienta de valor exploratorio para la investigación, y para sistematizar el resultado de estudios de caso mediante variables susceptibles de tratamiento multivariante con fines de modelización mediante la función discriminante (Plaza y Rufín 2005) o a través de modelos econométricos (Chen y Rossi 1987; Forés y Camisón 2008).

\subsection{El benchmarking como método de evaluación}

¿Qué lugar ocupa el benchmarking entre los métodos de evaluación? Inicialmente cabe afirmar su papel instrumental con valor exploratorio para la evaluabilidad del programa (Dahler-Larsen 2007) y analítico y confirmatorio para las hipótesis causales aunque, en ausencia de un método aceptado de contraste, los juicios que se derivan de la evaluación comparativa carecen en principio de valor de verdad, tal como se ha explicado.

Por el carácter abierto de la evaluación (Izquierdo 2008), el benchmarking se presenta entonces como un método de análisis aplicable a la práctica totalidad de los métodos aceptados 5 ; si bien, por sus características, aparece próximo a los enfoques del caso, del pluralismo cognitivo de Monnier (1995), orientado a la utilización de Patton (2004) y como respuesta a necesidades y apoyo a la toma de decisiones de Wholey (2004).

Con arreglo a su naturaleza de herramienta de gestión útil para la toma de decisiones y auxiliar para el análisis y determinación de la estrategia organizativa, se propone aquí añadir el benchmarking como categoría de Paraevaluación (cuasievaluación) a la tipología de Stufflebeam (200I) junto a las de Evaluación y Pseudoevaluación. De esta manera, el benchmarking no sería en sí mismo un método de evaluación por carecer de poder de inferencia; pero tampoco sería una falsa evaluación caracterizada por la presencia de elementos espurios, y sí un método cercano a la evaluación sistemática aceptada que, en

\footnotetext{
5 Con arreglo a los distintos criterios de clasificación: ex-ante/ex-post, por objetivos, para la rendición de cuentas, de impacto, de valor añadido, desempeño, coste/beneficio, coste/eficiencia y otras.
} 
los casos en los que la mera exposición de indicadores revelara evidencias, podría admitirse con valor de evaluación.

\subsection{Fiabilidad del benchmarking}

En cuanto que herramienta útil para conocer mejor la realidad investigada cabría preguntarse si la evaluación comparativa -ya como herramienta analítica ya evaluativa-, tiene requerimientos distintos para asegurar su fiabilidad frente a otros métodos de investigación.

Como sucede con los restantes métodos, la captura de la información necesaria es un proceso clave del que depende la calidad del resultado final del análisis. Por tanto, se requiere que los datos de partida de las distintas unidades objeto de comparación sean veraces, exactos y se expresen de forma homogénea.

Con frecuencia el uso del benchmarking forma parte de investigaciones realizadas por el método inductivo y, también en muchos casos, es habitual vincular la evaluación comparativa al análisis de casos y de mejores prácticas (Albors y Márquez 2008). En estas situaciones es evidente que no estamos ante procesos experimentales de investigación que requieran contar con muestras representativas.

Por el contrario, el sesgo que supone identificar y caracterizar casos particulares con los que comparar otros considerados unidades u objetos de estudio, es lo que hace de la evaluación comparativa un método útil para tal fin. A diferencia del método experimental en el que lo relevante es evitar sesgos que perjudiquen la representatividad de la muestra, en el caso del benchmarking lo relevante será, precisamente, introducir los criterios de sesgo idóneos para que como consecuencia del proceso de compa- ración, las diferencias entre el caso líder y los analizados resulten lo más nítidas posible; para poder investigar después la razón de tales diferencias y obtener conclusiones.

Dependiendo del ámbito temático, espacial, temporal y del nivel organizativo al que se plantee la investigación, es claro el papel que juegan los informantes clave en un proceso de benchmarking; en la medida que ayuden a definir con precisión los objetos y unidades de estudio, minimizando el riesgo de error.También, y como sucede con los restantes métodos de investigación, el análisis de una realidad cada vez más poliédrica requiere combinar distintas técnicas mediante procedimientos de Triangulación (Rodríguez 2005).

Por último, más que afirmar la fiabilidad del benchmarking como método de análisis y evaluación cabe señalar las cautelas necesarias que hacen de él un método fiable y que, como vemos, no difieren de las requeridas por cualquier otro método de investigación: calidad de la información de partida, adecuación del método, precisión en el enfoque, rigor en el análisis y triangulación.

\section{Perspectiva histórica del benchmarking}

La capacidad de objetivar que proporcionan los números y la universalidad de su lenguaje, hizo que a lo largo de los años cincuenta del siglo $X X$ el método comparativo basado en indicadores comenzara a aplicarse de manera sistemática. A partir de su primera utilización como método de análisis en ingeniería, y por extensión, comenzó a utilizarse también en el ámbito de las Organizaciones Internacionales aunque aplicado ya a la búsqueda de elementos homogéneos de comparación entre las políticas, planes, programas y proyectos que eran analizados (Cuadro I).

Cuadro I.

Benchmarking. Evolución histórica

\begin{tabular}{|c|l|}
\hline Período & \multicolumn{1}{c|}{ Función analítica preponderante } \\
\hline 1950-1975 & $\begin{array}{l}\text { Ingeniería inversa y competitividad empresarial. } \\
\text { Implantación en los Organismos Internacionales. }\end{array}$ \\
\hline 1976-1980 & Consultoría estratégica. \\
\hline $1981-1990$ & Conocimiento de la realidad social y organizativa. \\
\hline $1991-2000$ & Planificación por escenarios y prospectiva. \\
\hline $2001-2010$ & Análisis de políticas públicas. \\
\hline
\end{tabular}

Fuente: elaboración propia a partir de Keegan (1998). 
Desde entonces y hasta hoy, el benchmarking ha transitado por las cinco etapas que señala Keegan (1998) ampliando de forma progresiva su ámbito de aplicación o, si se prefiere, acentuando su transversalidad como método o herramienta de análisis.

En un primer período (1950-1975) la evaluación comparativa tuvo el carácter de ingeniería inversa y sirvió para analizar las características, funciones y prestaciones de productos o servicios competitivos entre sí. Con un enfoque de mercado, buscaba qué elementos del producto o servicio líder eran susceptibles de incorporarse a otros productos o servicios competidores.

En una segunda etapa, hasta 1980, el benchmarking se incorporó a la consultoría estratégica tomando como referencia lo exitoso de las experiencias de Xerox Corporation y Chaparral Stell (Cross 2000). En esta etapa, más que para evaluar programas públicos, la evaluación comparativa se constituyó como herramienta al servicio de lo que Porter (1996) denomina Eficiencia Operativa; esto es, desarrollar las actividades organizativas que proporcionan valor de una manera superior a la de los competidores. De esta forma, de lo que se trataba era de identificar qué actividades proporcionaban ventajas para la posición comercial en el caso de las empresas o de cara a la opinión pública en el caso de las instituciones.

Durante la década de los ochenta (1981-1990) el benchmarking traspasó las barreras sectoriales de la economía y la empresa para utilizarse (en esta tercera etapa) con fines de análisis para el conjunto de las ciencias sociales y ciencias de la dirección (management). De nuevo, fue la idea de proceso la que hizo de la evaluación comparativa una herramienta útil, vinculándola al análisis y gestión del cambio, del proceso directivo y del ciclo político.

En paralelo con la generalización de la planificación estratégica por escenarios, hasta el año 2000, el enfoque de la evaluación comparativa se hizo más amplio y profundo en su cuarta etapa, para convertirse en un proceso sistemático de análisis de escenarios alternativos, la puesta en práctica de estrategias y la mejora de la gestión.

La quinta etapa en la evolución de la evaluación comparativa alcanza hasta nuestros días, donde Subirats, Knoepfel, Larrue y Varor (2008) hacen del benchmarking referencia para el análisis profesional de las políticas públicas y de la interacción entre problemas sociales u organizativos. De la misma manera que en las economías competitivas el Sistema de Precios per- mite objetivar el valor relativo de intercambio, la proporcionalidad que reflejan los indicadores en un ejercicio de evaluación comparativa aplicado a las organizaciones, constituye para estos autores un elemento-guía como forma de evidenciar los costes y ventajas de una política o programa de acción.

Una vez más, la transversalidad del conocimiento permite que los fines que treinta años antes pretendiera el benchmarking de gestión, coincidan con los del análisis actual de las políticas públicas para estudiar e identificar qué factores determinan su éxito o fracaso. Además, se ponen de relieve «las similitudes y diferencias de los efectos que una misma política pública puede tener al implementarse en diferentes entidades públicas», así como «permitir que diversas administraciones compartan sus experiencias y enriquezcan sus procesos de aprendizaje», en expresión de los autores mencionados.

En consecuencia, los últimos diez años han sido testigos de la globalización del benchmarking. Su aplicación ha traspasado las fronteras de lo técnico, económico y social para abarcar también el análisis de las diferencias culturales en prácticamente todos los ámbitos de la actividad humana (Jordán y Pérez 2010).

Este hecho se ha visto también reflejado en el interés académico suscitado por la evaluación comparativa. Con un número anual medio de citas en torno a 18.000 entre 1990 y 2010 , desde el año 2000 las citas registradas por Google Académico de la voz «benchmarking» eran doble que las correspondientes a «SWOT» (Cuadro DAFO) y casi quintuplicaba las de «EFQM», por mencionar dos ejemplos de instrumentos de gestión conocidos y de uso común.

Como veremos más adelante, la conceptualización de los grandes desafíos de la Humanidad en los próximos años, obtendrá de la evaluación comparativa una colaboración eficaz a partir de su nueva dimensión global.

\section{Principales aplicaciones actuales del benchmarking}

El salto del benchmarking desde su nicho inicial de análisis de la competitividad empresarial, primero hacia el de la competitividad del sistema económico y después hacia el de la realidad social y organizativa, tiene que ver con la creciente necesidad por parte de los decisores (políticos, directivos y policy makers) de disponer de elementos objetivos sobre los que 
formular sus propuestas, llevarlas a la práctica y, finalmente, rendir cuentas ante sus electores de referencia; sean la ciudadanía, la junta de accionistas o el patronato en el caso de las organizaciones del Tercer Sector.

En buena medida, la adopción de la evaluación comparativa por un espectro tan amplio de ámbitos de gestión, se ha debido a la sencillez y gran poder de comunicación que reside tras una tabla de indicadores como producto final del análisis, y que nos recuerda el impacto que tuvieron en su momento la Tableau Économique de Quesnay y Turgot o Tabla Input-Output de Leontief.

Para precisar mejor el devenir de la evaluación comparativa en el transcurso de las últimas décadas, se han seleccionado cinco ámbitos sobre los que pivota en buena medida el progreso de las organizaciones de nuestro tiempo y en los que el benchmarking se ha aplicado con éxito: el conocimiento, la gestión de la información, la innovación, la gestión del talento y las políticas públicas.

\section{I. Conocer lo relevante}

Si un método de evaluación debe ser útil (Weiss 1973) tal utilidad deberá mostrarse por su capacidad para analizar la evaluabilidad del programa, clarificar la teoría, controlar los resultados y la utilización de los mismos.

Siguiendo a Keegan (2000a), a través de las modalidades de benchmarking holísitco y estratégico se han abordado evaluaciones cuyo fundamento residía en la utilización intensiva de fuentes secundarias de información estadística y cualitativa, lo ha que posibilitado evaluar programas de gran complejidad en forma modular como, por ejemplo, los llevados a cabo sobre el funcionamiento de los sistemas educativos ${ }^{6}$ o de salud ${ }^{7}$ en los ámbitos de la OCDE y la UE.

De esta manera ha sido posible abordar secuencialmente dimensiones que configuraban distintas situaciones-problema con economía de medios y priorizar las cuestiones estudiadas según su relevancia y nivel de riesgo, de acuerdo con los enfoques de Stake (1980) sobre el estudio de casos y la evaluación como respuesta responsable a las necesidades de los usuarios o clientes.
Por su parte, el benchmarking de diagnóstico se ha situado en línea con el planteamiento de Cronbach ( 1980 ) sobre la utilización de los resultados a corto plazo. Desde este punto de vista, la pluralidad de indicadores que permite utilizar la evaluación comparativa sirve para recoger los intereses de los diferentes stakeholders así como abordar otros contextos mediante la extrapolación del análisis.

\subsection{En la sociedad de la información}

En su faceta como herramienta informativa y de gestión de la información, la evaluación comparativa se ha utilizado para valorar las condiciones del entorno organizativo con carácter transversal (sincrónico) y longitudinal (diacrónico). Dichos marcos de análisis han permitido caracterizar la pertinencia y coherencia de los programas evaluados así como el ritmo o dinamismo con el que se desarrollaban asociándolo al nivel de recursos que requería la prestación de servicios.

También, y con carácter de paraevaluación, el benchmarking se ha utilizado en la evaluación de impacto y por resultados, vinculado generalmente a los criterios de cobertura, eficacia, eficiencia, equidad y sostenibilidad.

\subsection{Promover la innovación}

De acuerdo con su evolución histórica, lo que en sus inicios fuera un método innovador aplicado a la evaluación, se ha transformado en un recurso metodológico para evaluar la innovación, sus resultados e impacto.

En el ámbito de la organización industrial ha sido significativa la implantación del benchmarking para analizar la composición y funcionamiento de redes de innovación, determinar su coste, mecanismos de cooperación y eficacia de la transferencia de conocimiento entre los agentes participantes en dichas redes tales como consultores, empresas, centros de conocimiento y agencias públicas de desarrollo.

\subsection{Retener el talento}

Modernamente la gestión del talento y de los intangibles (Eustace 2000) aparece como uno de los cam-

\footnotetext{
6 Ver http://www.oecd.org/findDocument/0,3354,en_2649_37455_I_l_l_l_37455,00.html y http://ec.europa.eu/education/index_en.htm

7 Ver http://www.oecd.org/findDocument/0,3354,en_2649_37407_I_I_I_I_37407,00.html y http://ec.europa.eu/health-eu/index_en.htm
} 
pos en los que se utiliza con mayor intensidad la evaluación comparativa. En un mundo fragmentado por niveles de desarrollo y diferencias culturales y organizativas, es creciente la apelación a nuevos conceptos capaces de enfrentar la complejidad y gestionar mejor la interdependencia.

De nuevo, la comparación ha aparecido como recurso necesario para orientar la formación de polos o islas de excelencia en las que el conocimiento, la investigación sistemática y la internacionalización permitan alumbrar soluciones imaginativas.

En esta línea cabe señalar la extensión del benchmarking al funcionamiento del trabajo en red, las modalidades de cooperación público-privada, la educación universitaria y la formación de grupos de excelencia para llevar a cabo la investigación científica y tecnológica?.

\subsection{Promover la Reforma Regulatoria y modernizar la gestión de las organizaciones}

La evaluación de las políticas públicas es una disciplina científica que se justifica por la presión que experimentan las instituciones públicas para determinar en qué medida la intervención pública produce una mejora en el bienestar individual o social, cómo se produce esa mejora y cómo se podría conseguir de una forma más efectiva (Ballart 1996).

Si a comienzos de nuestro siglo el desafío de las sociedades europeas era adaptarse con éxito a la sociedad de la información (Liikanen 2000), diez años después el aumento de la capacidad innovadora de nuestras organizaciones y su ritmo para transformar la información en conocimiento aparecen como las palancas capaces de aumentar el valor del producto social.

Dichos cambios ha sido preciso abordarlos en un contexto de Reforma Regulatoria que orientara el sentido de las políticas públicas hacia esos fines (Balsa 2006). La evaluación comparativa está siendo utilizada como herramienta para identificar las mejores prácticas mundiales y aprender de ellas en lo que se refiere a la gestión del cambio, junto con el modo de acelerar la tasa de respuesta a las nuevas condiciones de entorno por parte de las organizaciones y gestores públicos y privados.
Hoy, el marco conceptual de la reforma de las regulaciones puede considerarse establecido en torno al concepto de «calidad de la regulación» que, aunque todavía impreciso, sirve para orientar dos principios básicos para su implementación. El primero de ellos es el de «regular bien antes que regular mucho» y, el segundo, «hacer medible la calidad regulatoria a través de indicadores útiles para sus destinatarios últimos»: gobiernos, expertos, grupos de interés y público en general.

Si en 1999 el benchmarking regulatorio se focalizaba en cómo simplificar el marco normativo para crear y facilitar el desarrollo de las unidades productivas (empresas), diez años después la prioridad se ha desplazado hacia campos tales como la estructura de las organizaciones, la generación y calidad del empleo, la gestión del presupuesto público, el e-gobierno y la medida de la integridad y seguridad jurídica de los países.

\section{Tendencias actuales del benchmarking para la evaluación organizativa y empresarial: el benchlearning}

Tras medio siglo de evolución teórica y metodológica, el benchmarking aplicado a la evaluación de las organizaciones y las políticas públicas o de empresa afronta cuatro retos. Los tres primeros se refieren a su capacidad para poner de relieve cómo mejorar la calidad de las regulaciones, cómo gestionarlas mejor y cómo comunicar los avances registrados; no sólo para los decisores sino también hacia la opinión pública. El cuarto desafío de la evaluación comparativa de hoy es su capacidad para identificar y caracterizar las mejores prácticas con fines de aprendizaje.

La mejora de la gestión regulatoria implica un cambio cultural que afecta a la totalidad del ciclo normativo y requiere de un esfuerzo continuado, a partir de establecer objetivos y prioridades de actuación en cuanto que programa de actuación destinado a incidir sobre gobiernos, organizaciones y empresas. En este contexto, la tendencia creciente es hacer del benchmarking una herramienta de aprendizaje (benchlearning) para la gestión del cambio en las organizaciones a través del intercambio de información, conocimiento y recursos (Garvin 2008, AEVAL 2009). A diferencia de la evaluación comparativa tradicional, el benchlearning refuerza la fase de análisis

\footnotetext{
9 En el ámbito europeo el Programa Marco de Investigación y Desarrollo Tecnológico se ocupa de estas cuestiones. Ver http://cordis.europa.eu/fp7/home_es.html
} 
con fines de aprendizaje, así como la identificación, selección y difusión de mejores prácticas (Cuadro 2).

De esta manera, la evaluación comparativa profesional orientada al aprendizaje requiere, en un primer momento, delimitar el fin exploratorio o confirmatorio de la investigación (Cuadro 3) y el nivel al que se realiza: individual (o de organización), sectorial (o de cluster) o de entorno (Cuadro 4).

Por su alcance, el benchlearning puede tener un enfoque estratégico o de posicionamiento organizativo o institucional a largo plazo. En el corto plazo, puede adoptar esquemas o prácticas vinculados a elementos más inmediatos de diagnóstico (Eykens 1997). Ambos enfoques introducen las posibles orientaciones alternativas de los trabajos (Cuadro 5).

\section{Conclusiones}

Tradicionalmente la evaluación comparativa ha servido para explicitar problemas, definir y ponderar los elementos constitutivos de las políticas o el funcionamiento de las organizaciones y empresas. En otros casos, se ha utilizado para mostrar la estructura de los recursos incorporados a la gestión valorando sus resultados e impactos. De forma añadida, los análisis anteriores han permitido formular hipótesis que, una vez contrastadas, han servido para establecer relaciones causales entre los efectos de los programas públicos y sus variables (drivers) determinantes.

A lo largo de este artículo se ha llevado a cabo una revisión crítica del benchmarking como método de paraevaluación o cuasievaluación, que ha situado en

Cuadro 2

Del benchmarking al benchlearning. Elementos diferenciales entre los procesos.

\begin{tabular}{|l|l|}
\hline \multicolumn{1}{|c|}{ Etapas del benchmarking } & \multicolumn{1}{|c|}{ Etapas del benchlearning } \\
\hline $\begin{array}{l}\text { I) Identificar los factores o elementos clave que caracterizan la } \\
\text { obtención de resultados superiores por una organización líder en } \\
\text { un escenario de referencia. }\end{array}$ & $\begin{array}{l}\text { I) Identificar los factores o elementos clave que caracterizan la } \\
\text { obtención de resultados superiores por una organización líder en } \\
\text { un escenario de referencia. Identificación de un marco de apren- } \\
\text { dizaje. }\end{array}$ \\
\hline $\begin{array}{l}\text { 2) Analizar e identificar indicadores representativos de las varia- } \\
\text { bles o factores clave para la organización líder en el escenario de } \\
\text { referencia. }\end{array}$ & $\begin{array}{l}\text { 2) Analizar e identificar indicadores representativos de las varia- } \\
\text { bles o factores clave para la organización líder en el escenario de } \\
\text { referencia. Contextualizar la viabilidad y posibilidad de aprendiza- } \\
\text { je por las organizaciones comparadas. }\end{array}$ \\
\hline $\begin{array}{l}\text { 3) Investigar por qué la organización líder en el escenario de re- } \\
\text { ferencia obtiene resultados ventajosos sobre las demás. }\end{array}$ & $\begin{array}{l}\text { 3) Investigar por qué la organización líder en el escenario de re- } \\
\text { ferencia obtiene resultados ventajosos sobre las demás. Definir y } \\
\text { estructurar procesos de aprendizaje ad hoc. }\end{array}$ \\
\hline $\begin{array}{l}\text { 4) Establecery aplicar planes de acción en las organizaciones com- } \\
\text { paradas a través de los indicadores con potencial de mejora con } \\
\text { respecto a los de la organización líder. }\end{array}$ & $\begin{array}{l}\text { 4) Elaborar estudios de caso, planes formativos y actividades de } \\
\text { difusión hacia las organizaciones comparadas en las áreas que pre- } \\
\text { senten potencial de aprendizaje y mejora. Desarrollar acciones de } \\
\text { coaching, tutoría o mentoring. }\end{array}$ \\
\hline
\end{tabular}

Fuente: elaboración propia.

Cuadro 3

Benchmarking. Naturaleza de las investigaciones.

\begin{tabular}{|l|l|}
\hline \multicolumn{1}{|c|}{ Modalidad } & \multicolumn{1}{c|}{ Aplicaciones } \\
\hline Exploratoria o predictiva & $\begin{array}{l}\text { Para identificar variables potencialmente relevantes y establecer } \\
\text { sobre ellas hipótesis causales susceptibles de contraste. }\end{array}$ \\
\hline Confirmatoria o de verificación & $\begin{array}{l}\text { A partir de variables cuya relación causal haya sido probada, la in- } \\
\text { vestigación se orienta a verificar su validez en nuevos contextos } \\
\text { transversales (estudios cross section) o temporales (estudios lon- } \\
\text { gitudinales). }\end{array}$ \\
\hline
\end{tabular}

Fuente: elaboración propia. 
Cuadro 4

Modalidades y niveles de benchlearning.

\begin{tabular}{|l|l|l|}
\hline \multicolumn{1}{|c|}{ Modalidad } & \multicolumn{1}{|c|}{ Nivel estratégico } & \multicolumn{1}{c|}{ Nivel de diagnóstico } \\
\hline Exploratorio o predictivo & $\begin{array}{l}\text { Individual o de organización } \\
\text { Sectorial o de cluster } \\
\text { De escenario o entorno }\end{array}$ & $\begin{array}{l}\text { Individual o de organización } \\
\text { Sectorial o de cluster } \\
\text { De escenario o entorno }\end{array}$ \\
\hline Confirmatorio o de verificación & $\begin{array}{l}\text { Individual o de organización } \\
\text { Sectorial o de cluster } \\
\text { De escenario o entorno }\end{array}$ & $\begin{array}{l}\text { Individual o de organización } \\
\text { Sectorial o de cluster } \\
\text { De escenario o entorno }\end{array}$ \\
\hline
\end{tabular}

Fuente: elaboración propia.

Cuadro 5

Niveles de benchlearning y orientación de los trabajos

\begin{tabular}{|c|c|}
\hline Nivel & Orientación \\
\hline Individual o de organización & $\begin{array}{l}\text { Como herramienta destinada a medir la capacidad de mejora y aprendizaje continuo en pro- } \\
\text { cesos de gestión de la innovación, del desarrollo organizativo y de la gestión de recursos in- } \\
\text { formativos, humanos, materiales y otros. } \\
\text { Su alcance puede ser la búsqueda del posicionamiento de la organización a medio y largo pla- } \\
\text { zo en el concierto institucional (modalidad estratégica) o para desarrollar técnicas de gestión } \\
\text { operativa vinculadas a procesos clave de la organización (modalidad de diagnóstico). }\end{array}$ \\
\hline Sectorial o de cluster & $\begin{array}{l}\text { Constituye la extensión lógica de la aplicación del benchmarking individual para estudiar prác- } \\
\text { ticas organizativas en el concierto sectorial: principios de actuación, problemas comunes e iden- } \\
\text { tificación de mejores prácticas. } \\
\text { Su alcance puede ser de posicionamiento a largo plazo en el sector de actividad (modalidad } \\
\text { estratégica) o para desarrollar técnicas operativas de gestión (modalidad de diagnóstico). }\end{array}$ \\
\hline Condiciones de escenario o entorno & $\begin{array}{l}\text { El alcance del análisis rebasa en este nivel los ámbitos sectorial e individual y se aplica al estu- } \\
\text { dio de los elementos clave que afectan al atractivo cultural, social y económico de un territo- } \\
\text { rio (ciudad, región o país) desde el punto de vista de las condiciones de entorno necesarias } \\
\text { para abordar su participación en programas de desarrollo amplios y complejos. } \\
\text { En este nivel las condiciones culturales, sociales, regulatorias, económicas y de dotación de in- } \\
\text { fraestructuras se presentan interrelacionadas en las escalas local, regional, nacional y suprana- } \\
\text { cional. } \\
\text { Las materias objeto de análisis se refieren a macroprocesos de orden cultural, empresarial, eco- } \\
\text { nómico, político y de relaciones internacionales (procesos cooperativos, prevención de crisis, } \\
\text { gestión de conflictos y otros). } \\
\text { Aunque su alcance suele ser el posicionamiento a largo plazo (modalidad estratégica) pueden } \\
\text { evaluarse también procedimientos de gestión a más corto plazo (modalidad de diagnóstico) } \\
\text { para escenarios concretos. }\end{array}$ \\
\hline
\end{tabular}

Fuente: elaboración propia.

paralelo la robustez que ofrece este instrumento analítico y su poder de comunicación, junto con las limitaciones de orden espistemológico que presenta.

En el nuevo escenario que configuran problemas y desafíos globales, la capacidad para objetivarlos que caracteriza al benchmarking previsiblemente va a verse enriquecida con los avances en la modelización de tales escenarios complejos orientada al aprendizaje de las mejores prácticas (benchleraning).

El trabajo de Stiglitz, Sen y Fitoutssi (2009) ha venido a recordar las limitaciones que muestran los indicadores tradicionales para medir el progreso social y el crecimiento económico. Por tanto, se impone una visión sistémica de los problemas que necesa- 
riamente debe ir acompañada del análisis conjunto de las variables que mejor puedan caracterizarlos primero, para señalar después cuáles son los resortes sobre los que actuar y cuál debe ser la relación entre ellos, de forma que se incremente la eficacia de las políticas que los gestionen.

En cada situación la lógica del análisis señalará la existencia de un caso que, por sus características, pueda ser tomado como referencia para referir a él los parámetros analizados y, consecuentemente, aparecerá la evaluación comparativa como herramienta de uso inmediato susceptible de formar parte de esquemas metodológicos de distinta complejidad. Quizá sea esa característica de inmediatez la que haga previsible el uso sostenido del benchmarking como método analítico y evaluativo en los próximos años. Sin pretender agotar lo que sería un catálogo de grandes cuestiones en las que el benchmarking puede aportar la capacidad de análisis sistémico mencionada, se citan:

- Superar la medida de la riqueza y el progreso a través de los indicadores tradicionales mediante la definición de otros complementarios a la hora representar la demanda social en torno a conceptos tales como Bienestar, Felicidad, Goberanza y Responsabilidad, entre otros.

- Abordar la virtualización institucional y organizativa del Gobierno, la Universidad y la Empresa como consecuencia de Internet y las posibilidades que ofrecen las tecnologías de la información.

- Caracterizar la creación de redes y plataformas de excelencia en torno al conocimiento, la innovación y las tecnologías de las comunicaciones, tales como las desarrolladas en torno a los conceptos y herramientas web 2.0.

- Gestionar desafíos tales como la búsqueda de nuevas modalidades de empleo, la lucha contra la pobreza, el cambio climático y la seguridad colectiva.

El benchmarking actual no deja de ser una aplicación más perfecta y modelada por la experiencia de siglos de la máxima de: «comparar para aprender y mejorar», y así se presenta hoy. Casi medio siglo después de que la necesidad llevara a la compañía Xerox a formalizar el uso de esta herramienta para explicar el porqué de unos resultados económicos decepcionantes, la voz benchmarking registra más de nueve millones de referencias en Google, existen sociedades profesionales que perfeccionan su uso y se emplea habitualmente con fines de investigación y análisis en multitud de ámbitos sociales, académicos y políticos.
La exhortación de Rousseau al analizar los signos de un buen gobierno continúa siendo actual aplicada a los gobiernos, las organizaciones y las empresas: «calculadores, ahora el asunto es vuestro, contad, medid, comparad».

\section{Bibliografía}

AGENCIA DE EVALUACIÓN Y CALIDAD (AEVAL) (2009): Modelo EFQM de excelencia adaptado a la administración pública. Manual de autoevaluación. Madrid, AEVAL.

ALBORS, J., MÁRQUEZ, P. (2008): «Las relaciones fabricante distribuidor como elementos básicos de competitividad: evaluación de tres factores moderadores. Análisis empírico en el caso del cluster cerámico español». Dirección y organización n. ⒊ 35, pp. 79-87.

ALLEN, D. ( 1996): «Benchmarking». Diario ABC. Madrid, I de septiembre de 1996.

BALSA, C. (2006): La «better regulation». Papeles de Evaluación. Agencia de Evaluación y Calidad (AEVAL).

BALLART, X. (1996): «Modelos teóricos para la práctica de la evaluación». Lecturas de gestión pública. Madrid, Instituto Nacional de Administración Pública (INAP), pp. $321-352$.

BELTRÁN, M. (1985): «Cinco vías de acceso a la realidad social». Revista Española de Sociología (REIS) n. ${ }^{\circ} 29$, pp. 7-42.

BOGAN, C., ENGLISH, M. (1994): Benchmarking for best practices: Winning through innovative adaptation. Nueva York, McGraw-Hill.

BOXWELL, R. (1994): Benchmarking for a competitive advantage. Nueva York, McGraw-Hill.

CAMP, R. (1989): The search for industry best practices that lead to superior performance. ASQ Quality Press.

CAMP, R. (2000): «The path to excellence. Benchmarking in Europe». PSI Group, Primavera de 2000, pp. I4- 17.

CHEN, H., y ROSSI, P. ( I 987): «The theory-driven approach to validity». Evaluation and program planning, 10 , pp. 95- 103.

COMISIÓN EUROPEA (2000a): Benchmarking enterprise policy. Bruselas, 29 de febrero.

COMISIÓN EUROPEA (2000b): «Commission staff working paper. Benchmarking enterprise policy: an outline for the work ahead». Documento SEC(2000) 821 . Bruselas, II de mayo.

COMISIÓN EUROPEA (2003): Benchmarking business angels. Best report n. ${ }^{\circ}$ I. Bruselas. 
CRONBACH, L. et al (1980): Towards reform of Program Evaluation. San Francisco, CA, Jossey-Bass.

CROSS, R. (2000): «The Xerox experience». Benchmarking in Europe. PSI Group, Primavera, pp. 38-39.

DAHLER-LARSEN, P. (2007): «iDebemos evaluarlo todo? O de la estimación de la evaluabilidad a la cultura de la evaluación». Información Comercial Española (ICE), n836, pp.93-104.

DENRELL, J. (2003): Selection bias and the perils of benchmarking. Harvard Business School Publishing Corporation.

DRUCKER, P. (1986): «La disciplina de la innovación». Harvard-Deusto Business Review, $2^{\circ}$ trimestre.

EUSTACE, C. (2000): The intangible economy impact and policy issues. Bruselas, Comisión Europea.

EYKENS, M. et al (1997): ¿Cómo puede Europa prevenir los conflictos? The Phillip Morris Institute for Public Policy Research.

FORÉS, B., y CAMISÓN, C. (2008): «La capacidad de absorción de conocimiento: factores determinantes internos y externos». Dirección y Organización, n 36, pp. 35-50.

GARVIN, D. (2008): Building a learning organization. Harvard Business School Publishing Corporation.

HIDALGO, A., VIZÁN, A., TORRES, M. (2008): «Los factores clave de la innovación tecnológica: claves de competitividad empresarial». Dirección y organización n. ${ }^{\circ} 36$, pp. 5-22

INTXAURBURU, M., y OCHOA, C. (2005): «La herramienta de benchmarking: iestrategia de imitación o innovación?». IX Congreso de ingeniería de organización. Gijón.

IZQUIERDO, B. (2008): «De la evaluación clásica a la evaluación pluralista. Criterios para clasificar los distintos tipos de evaluación». Empiria. Revista de Metodología de Ciencias Sociales, n. ${ }^{\circ}$ 16, pp. II 5-134.

JORDÁN, A., PÉREZ, J. (20 I0): «Metodología para el análisis de la relación entre la competitividad y la preparación tecnológica en I 35 economías mundiales. Factores e indicadores clave de actuación. Conglomerados». Dirección y organización n. ${ }^{\circ}$ 40, pp. 28-47.

KEEGAN R. (1988): Benchmarking facts. A European perspective. Dublin, Comisión Europea, DG-III-Industria.

KEEGAN R. $\left(2000^{\mathrm{a}}\right)$ : «Supporting benchmarking of enterprises». Enterprise Ireland. Concerted Action Seminar. Atenas, 15-16 de mayo.

KEEGAN, R. (2000b): «The European benchmarking forum». Benchmarking in Europe, PSI-Group, primavera, pp. 64-65.
MARTíNEZ, A. (1993): Metodología de las ciencias. Madrid, Universidad Nacional de Educación a Distancia (UNED).

McKENZIE,W. ( 1 972): Política y ciencia social. Madrid, Aguilar.

McLEAN, I ( 1996): The concise dictionary of politics. Oxford University Press.

MOLERO, J. (200 I): Innovación tecnológica y competitividad en Europa. Madrid, Síntesis.

MONNIER, E. (1995): Evaluación de la acción de los poderes públicos. Madrid, Instituto de Estudios Fiscales (Ministerio de Economía y Hacienda), $2^{a}$ edición.

ORGANIZACIÓN PARA LA COOPERACIÓNY EL DESARROLLO ECONÓMICO (OCDE) (2005): Micro-policies for growth and productivity. Syntesis and benchmarking user guide. París.

ORGANIZACIÓN PARA LA COOPERACIÓNY EL DESARROLLO ECONÓMICO (OCDE) (20I0): Ministerial conclusions. Meeting of the council. París, 27-28 de mayo.

PATTON, M. (2004): «The roots of utilization-focused evaluation». Evaluation roots. Tracing theorist's views and influences. M. Alkin, compilator. Sage.

PETERS, T. (2002): El talento, cómo identificarlo y mantenerlo. Nowtilus.

PLAZA, J., y RUFÍN, R. (2005): «Un análisis de la influencia combinada de la capacidad comercial y de las tecnologías informativas (TIC) sobre las PYME españolas innovadoras». Estadística española, vol. 47, n. I60, pp. 50 I-537.

PORTER, M. (1996): What is strategy? Harvard Business School Publishing Corporation.

RODRÍGUEZ, O. (2005): «La triangulación como estrategia de investigación en ciencias sociales». Revista de Investigación en Gestión de la Innovación y la Tecnología $(\mathrm{mi}+\mathrm{d}), \mathrm{n}^{\circ} 31$

ROSSI, P., y FREEMAN, H. (1989): Evaluation. A Systematic Approach. Beverly Hills, CA, Sage.

SCRIVEN, M. (1980):The logic of evaluation. Inverness, CA, Edgepress.

SPENDOLINI, M. ( 1992): Benchmarking. Nueva York, Amacom (traducido al español en 1994 por la Editorial Norma).

STAKE, R. (1980): «Program evaluation, particularly responsive evaluation». En Rethinking Educational Research. London, Hodder \& Stougton, pp. 72-87.

STIGLITZ, J., SEN, A., y FITOUSSI, J. (2009): Report by the Commission on the measurement of economic performance and social progress. París. 
STUFFLEBEAM, D. (200 I): Evaluation models. New directions for American, Evaluation, Association, n. ${ }^{\circ} 89$.

SUBIRATS, J., KNOEPFEL, P., LARRUE, C., y VAROR, F. (2008): Análisis y gestión de políticas públicas. Barcelona, Ariel.

WATSON, G. (1993): Strategic benchmarking: How to rate your company's performance against the world's best. Nueva York, John Wiley \& Sons.
WEISS, C. (1973): «The politics of impact measurement». Policy studies Journal, n. ${ }^{\circ}$ I, pp- I79-I 83.

WHOLEY, J. (2004): «Using evaluation to improve performance and support policy decision making». Evaluation roots. Tracing theorist's views and influences. M. Alkin, compilator. Sage. 